\title{
Sentinel lymph node in endometrial cancer: an overview
}

\author{
Elisabete Gonçalves • Odete Figueiredo • Fernanda Costa
}

Received: 22 January 2013 /Accepted: 11 April 2013 /Published online: 7 May 2013

(C) Springer-Verlag Berlin Heidelberg 2013

\begin{abstract}
The role of complete pelvic and para-aortic lymphadenectomy in early endometrial cancer remains controversial in gynecologic oncology. Sentinel lymph node detection is an alternative to assess lymphatic spread in several solid tumors. The authors review the literature related to the detection of sentinel lymph node in endometrial cancer, the techniques employed, and its results and feasibility. The authors review reported case series of endometrial cancer in which the sentinel lymph node biopsy was performed. A systematic literature review was conducted using the PubMed database. Different techniques were used considering lymphatic imaging mapping (colorimetric, isotopic, and fluorescence procedures) and injection site (subserous, hysteroscopic, and cervical). Detection rates of sentinel lymph node were heterogeneous, varying between 44 and $100 \%$ with false-negative rates between 0 and $33 \%$. Although technically demanding, hysteroscopy approach was associated with the highest detection rate. The largest trials showed a good detection rate with cervical injection, a more reproducible procedure. The laparoscopic route improved the results. Immunohistochemistry staining improved the micrometastasis detection in sentinel lymph node. Cost-effectiveness of systematic lymphadenectomy compared with sentinel lymph node procedure and its value on adjuvant therapies as well as a standardized reproducible and reliable technique must be assessed.
\end{abstract}

Keywords Sentinel lymph node · Endometrial cancer · Lymph node mapping $\cdot$ Ultrastaging

\footnotetext{
E. Gonçalves $(\bowtie) \cdot$ O. Figueiredo $\cdot$ F. Costa

Division of Gynecology Oncology, Department of Gynecology and Obstetrics of Centro Hospitalar Tâmega e Sousa, Lugar do Tapadinho, Guilhufe, 4564-007 Penafiel, Portugal

e-mail: elisabetegoncalvesms@yahoo.com
}

\section{Background}

Endometrial cancer (EC) represents the most frequent malignancy of the female genital tract in developed countries. In 2011, 46,470 new cases of EC were diagnosed and 8,120 EC-related deaths occurred in the USA [1]. Seventy percent are diagnosed at an early stage [2] and are treatable by surgical intervention.

EC is surgically staged using the 2009 International Federation of Gynecology and Obstetrics (FIGO) staging system. Surgical treatment consists of peritoneal washing, total hysterectomy, bilateral salpingo-oophorectomy, and pelvic and para-aortic (PA) lymphadenectomy [2].

The risk of metastatic involvement of lymph nodes is dependent on histological type, grade, depth of myometrial invasion, and lymphovascular invasion [3, 19]. Nevertheless, the risk of metastatic disease to pelvic lymph nodes at initial stages (IA grade 1-2) is $0-7 \%$ associated to a low risk of recurrence (3-6\%) [3]. On the other hand, advanced stages and grade 3 endometrial cancer are associated with a lymph node metastatic involvement as high as $20 \%$ [3]. When pelvic nodes are positive, PA nodal involvement occurs approximately in $50 \%$, and in up to $6 \%$ of patients, it may occur isolated [4]. Therefore, in 80-90\% of early stage endometrial cancer, pelvic and PA lymphadenectomy is useless, incurring morbidity [3].

Survival impact of systematic lymphadenectomy in patients with EC is widely discussed. The ASTEC trial shows no evidence of benefit for systematic lymphadenectomy for early EC in terms of overall and recurrence-free survival [5].

The sentinel lymph node (SLN), by definition, is the first node to receive metastasizing cancer cells, as first described in 1960 by Gold et al. for parotid cancer [6]. In 1996, Burke et al. reported the SLN first application in EC [7].

Nowadays, lymphatic mapping is an accepted alternative to assess lymphatic spread in several solid tumors. EC 
lymphatic mapping has not gained the same popularity. The uterus has a complex lymphatic drainage due to lymphatic channels in the broad and infundibulo-pelvic ligaments [7]; therefore, the lymphatic spread is through external iliac and obturator (mainly) and PA area, respectively (Fig. 1), and the best technique to detect SLN is yet unestablished [7].

First studies of SLN in EC included small-size samples and various injections sites revealing heterogeneous results. In 2008, a consensus panel stated that SLN in EC was worthy of further investigation [8]. Prospective investigation is reviving SLN in EC.

EC patients are usually obese and aged [9]. The primary goal of SLN in EC consists in reducing the morbidity of a full lymphadenectomy (by decreasing surgical time), as well as early (need of blood transfusion, fever, wound infection, thromboembolism [10]) and late complications (lymphocysts formation, leg lymphedema, transient neuralgias [3, 6, 8-14]). Besides, SLN allows an accurate exploration of all drainage routes as well as those considered aberrant or not routinely examined [12]. It is suggested that SLN could have a value in isolated PA metastatic node detection. PA metastasis is a

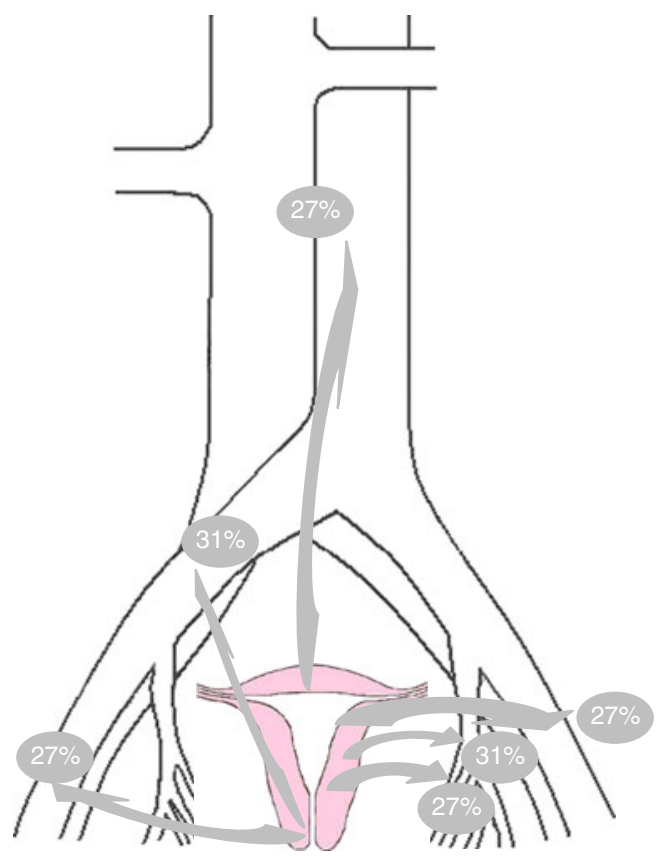

Fig. 1 Main routes of lymphatic drainage and relative distribution of lymphatic nodes in EC. The concept of SLN depends on the uterus's lymphatic drainage. There are two main interconnected routes through broad and infundibulo-pelvic ligaments, these last ones considered the secondary routes. The cervix and the lower uterine segment drain mainly through parametria to external iliac and common iliac nodes. The uterine corpus drains primarily to the external iliac nodes; other pathways include the inter iliac, common iliac, and obturator area. Uterine fundal drainage is relatively consistent through the ovarian vessels to the infra-renal PA area known adverse prognostic factor in EC [15], and there is no reliable way to predict its involvement [16]. SLN detection could select patients that beneficiate from PA lymphadenectomy that would be performed in presence of a positive SLN. For this purpose, the most accurate technique involves hysteroscopic injection [17]. Moreover, the use of more sensitive techniques for SLN detection could better identify pelvic nodes and probably reduce the reported incidence of isolated infra-renal metastasis [8]. The secondary goal is to improve detection of micrometastasis, which is associated with a recurrence risk [13], with resource to ultra-sectioning and immunohistochemestry (IHC) protocols [3, 6, 8-13]. Therefore, the risk of recurrence related to positive lymph nodes missing despite a full lymphadenectomy could be reduced [12], and SLN could improve selection of patients for adjuvant therapy. Despite the controversy concerning therapeutic benefit of lymphadenectomy, some experts argue that lymph node staging is necessary to guide appropriate adjuvant therapy [18]. Adjuvant therapies based only on uterine factors may result in over or under treatment $[12,32]$. Identification of microscopic disease outside the uterus can help to determine the need of adjuvant therapy. SLN application in EC is more accurate than MRI and intra-operative frozen section analysis in assessing lymph node status [1,8] and SLNs are three times more likely to harbor disease than non-SLNs [34]. SLN in EC, incorporating ultrastaging, may improve selection of women at high risk and could potentially avoid $80 \%$ full unnecessary lymphadenectomy [6]. The authors review the concerning techniques employed and their results and feasibilities to detect SLN node in EC.

\section{Material and methods}

The authors performed a comprehensive and systematic search on the PubMed database for published studies with keywords "sentinel lymph nodes," "endometrial cancer," "lymphadenectomy," and "ultrastaging". We selected studies evaluating techniques to detect SLN including original articles, meta-analysis, reviews, and opinion articles published up to October 2012.

Authors analyzed lymphatic mapping methodology, injection site, surgical route, and detection of micrometastasis, of which advantages and disadvantages, rates of detection of SLN, and negative prognostic values were correlated.

\section{Findings}

Twenty-three publications with at least 15 cases were included. Eighteen studies were prospective, and one was a 
meta-analysis involving 26 studies. Four studies analyzed subserosal injection, five hysteroscopic injection, and one peri-tumoral ultrasound guided injection. Eleven analyzed cervical injection. One evaluated cervical and subserosal injections simultaneously. The meta-analysis intended to assess the diagnostic performance of SLN through univariate regression analysis. The characteristics of these studies are summarized in Table 1.

SLN detection varies depending on the lymphatic mapping procedure, injection site, and surgical route [9]. Other factors include surgeon experience, delay between injection and detection, and previous pelvic radiotherapy or surgery.

\section{Lymphatic mapping procedure}

The detection of SLN is performed using colorimetric imaging of blue dyes (isosulfan blue, patent blue, and methylene blue), isotopic mapping with Technetium-99 (Tc-99), or both, through injection in a defined location [3, 8, 9]. Half-life is relatively long to allow a deferred identification of SLN, and both have a safety profile [9]. Blue dye imaging is injected before surgery, after induction of anesthesia [11]. Isotopic imaging implies an injection, ideally $18 \mathrm{~h}$ before surgery, with control scintigraphic images obtained with a gamma camera.

During surgery, pelvic and lower PA regions are inspected for colored lymph node channels and dye uptake. Radioactive pelvic and PA lymph nodes are localized using a gamma probe (usually before opening the retroperitoneum). Dissection of pelvic lymph nodes with or without PA management is performed and followed by hysterectomy with bilateral salpingo-oophorectomy.

Dual detection (colorimetric and isotopic) was associated with the highest detection rate and lowest false-negative rate, independent of injection site [9, 22], with detection rates ranging from 46 to $87 \%$ [3]. Ballester et al. demonstrated a SLN detection rate of $57 \%$ with simple colorimetric imaging against $94 \%$ in double detection, using cervical injection [23]. Later, Ballester et al. found low correlation between day-before lymphoscintigraphy and surgical SLN mapping querying its usefulness and cost-effectiveness in routine practice [24].

Solima et al. found a high detection rate of SLN when performed by hysteroscopic injection of radiolabeled albumin colloids and suggested that this result may be explained by the low interval between hysteroscopic injection and sentinel node detection, not exceeding $6 \mathrm{~h}$ [21]. Kang et al. found no statistical difference in a meta-analysis when comparing dye, isotope, or both [20].

Other medical dyes, such as Indocyanine green (ICG) with near-infrared fluorescence, have recently been reported for use in mapping $[8,22]$. Roy et al. applied robotically assisted fluorescence imaging with ICG for lymphatic mapping in early stage cervical and endometrial cancer reporting a $7.8 \%$ increase in SLN detection $[8,22]$. Holloway et al. compared, in a prospective study with 35 women with EC, colorimetric and fluorescence imaging. Bilateral SLNs were detected in $97 \%$ using the robotic near-infrared imaging system and in $77 \%$ patients by colorimetric analysis [8]. Holloway et al. found this technique easy to perform and complimentary to traditional calorimetric imaging. If these findings are corroborated in the future with larger multicentric trials, Holloway et al. argue that gynecologist oncologists will be more confident omitting lymphadenectomy for low-risk EC by performing less morbid SLN biopsies and will improve the precision of staging for high-risk EC [8].

Injection site

Considering the complexity of uterus lymphatic drainage [7,9], the injection site is the most evaluated issue for SLN detection. Despite being a major determinant [3], the optimal site remains unclear [14]. Different injection sites were experimented, and combined techniques were also proposed $[3,9,14]$.

\section{Subserosal/myometrial injection}

This was the first procedure used for SLN detection in EC, as published by Burke et al. The 15 patients involved underwent laparotomy, and after obtaining peritoneal washings, the uterus was exposed and the fallopian tubes were occluded; blue dye was injected into subserosal myometrium at three midline places (empirical-midline injections were used to maximize the probability of observing bilateral lymphatic drainage). The colored lymphatic channels were dissected in pelvic and PA regions followed by standard surgical staging [7]. They reported a SLN detection rate of $67 \%$ with a false-negative rate of $50 \%[3,7,9,25]$. Other seven articles describe this technique (Table 1). Detection rate ranges considerably between 0 and $92 \%$ with sensibilities spanning 50 $100 \%$. False-negative range is $0-50 \%$, and the negative predictive value is $75-100 \%$. Altgassen et al. reported the highest detection rate $(92 \%)$, which was related with the higher number of myometrial injections performed (eight) [25]. Moreover, adding to the safety and feasibility, this technique has a good accuracy to detect PA lymph nodes with a rate of detection of 31-34\%. Disadvantages attributed to this technique include surgical planning difficulties and required dissection of all anatomical areas of lymphatic drainage [25].

\section{Peri-tumoral/hysteroscopic injection}

The hysteroscopic injection is technically the most difficult, mainly because of direct access to the injection site $[3,9,14$, 27]. Success rate is reported as high as 64-100\% [9]. Disadvantages include eventual local anesthesia or sedation for good tolerance and the complex logistics of performing 


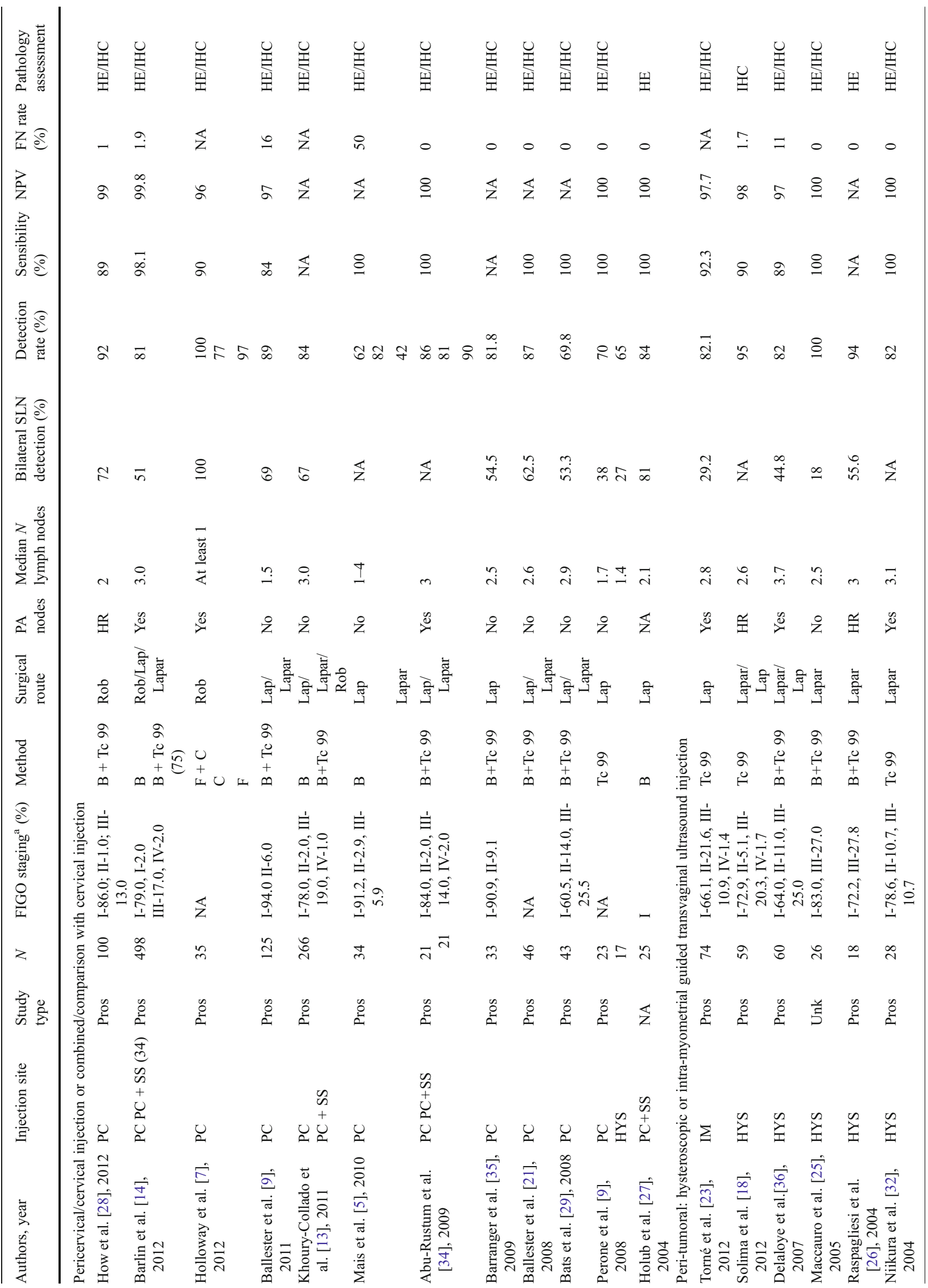




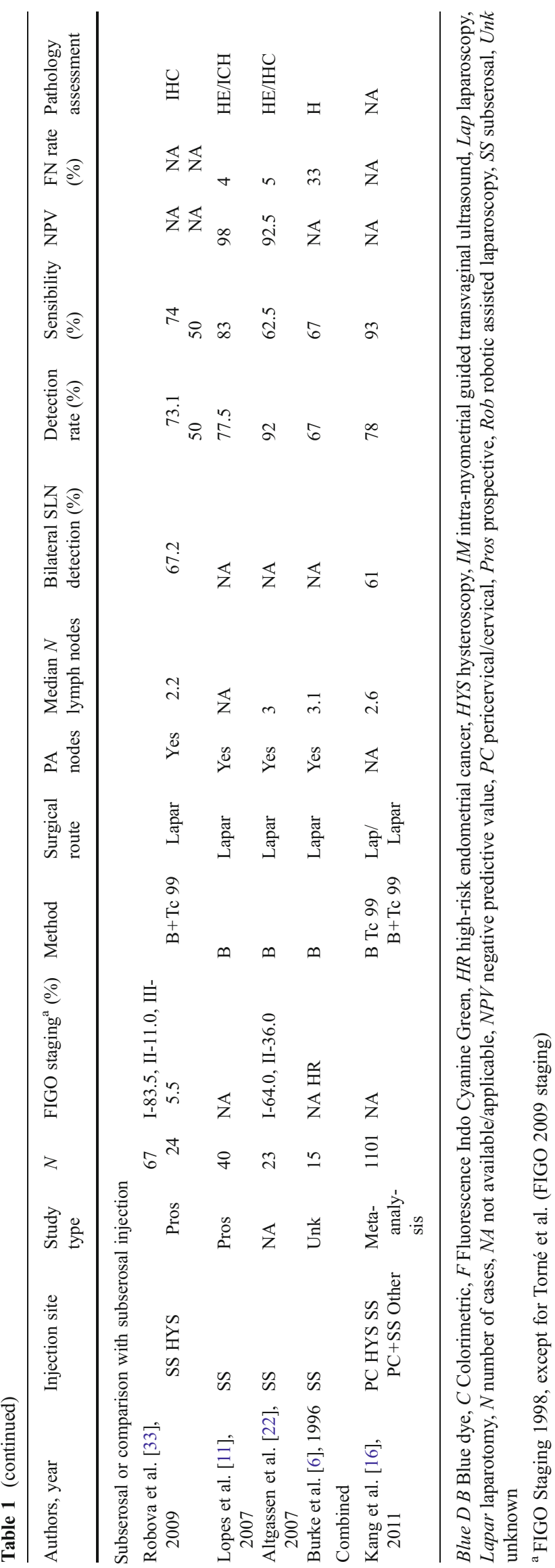

the injection per-operatively, considering that the use of radioactive agents is not allowed in the conventional operating theater $[9,27]$. Another issue raised concerns with the repeated hysteroscopy that could favor the dissemination of tumor cells through the fallopian tubes to the peritoneal cavity [3, 9, 27]. Maccauro et al. and Raspagliesi et al. reported one case of positive peritoneal cytology [28, 29]. However, the dissemination risk is low if less than $70 \mathrm{mmHg}$ is used to distend uterine cavity [28]. Gien et al. evaluate peritoneal washings after hysteroscopy and found no positive cytology for malignancy [14].

Niikura et al. suggested that with hysteroscopic guidance and the possibility to determine the injection point into the endometrium, the isotope pathway will mimic the natural lymphatic drainage of cancer cells [28]. It is associated with the highest reported SLN detection rates [28], ranging between 40 and $100 \%$, and it detects PA lymph nodes with rates as high as $57 \%[3,9,19,28]$.

Raspgaliesi et al. and Maccauro et al., in their series with 18 and 26 patients, respectively, injected a radioactive tracer and blue dye by hysteroscopy and performed surgery $3-4 \mathrm{~h}$ after. They found a $100 \%$ SLN detection, and all metastatic nodes were identified as SLN [14, 28, 29].

Solima et al. published in 2012 the largest serial with 80 patients using office hysteroscopic technetium injection (office hysteroscopy was performed without cervical dilation or local/general anesthesia, with maximum intracavitary pressure of $40 \mathrm{mmHg}$ ). Radiolabeled albumin $99 \mathrm{Tc}$ was injected peritumoral at 3, 6, 9, and $12 \mathrm{~h}$ subendometrially; if the entire cavity was involved, technetium was injected at four uterine surfaces and the fundus. Gamma camera images were obtained starting $15 \mathrm{~min}$ after injection, every $5 \mathrm{~min}$ in $1 \mathrm{~h}$. Pelvic and PA lymphadenectomies were performed systematically in EC serous or clear cell histological types, and for endometrioid type when intraoperative staging was equal or higher than IB G2. They reported at least one SLN in 76 of the 80 patients. Ten of these patients $(17 \%)$ had node metastases. Thirty-three patients (56\%) had SLN in the PA region. Negative predictive value (NPV) was $98 \%$ (95\% CI 89.4 $100)$ and sensitivity $90 \%$ (55.5-99.8). Solima et al. related the SLN detection rate with the low interval between hysteroscopic injection and surgery, up to $6 \mathrm{~h} \mathrm{[21].}$

Lately, a new approach for SLN detection in EC was proposed by Torné et al.: transvaginal ultrasound-guided myometrial injection of radiotracer (TUMIR) consists in a myometrial injection of radiotracer guided by trans-vaginal ultrasound resembling the embryo transfer procedure. The protocol procedure described by Torné et al. used a Tc 99 injection in the anterior and posterior myometrium wall, guided by ultrasound and performed under local anesthesia, 18-24 h before surgery. Identification of SLN was performed with lymphoscintigraphy preoperatively. Patients underwent laparoscopy, and radioactive pelvic and PA SLNs were 
identified with a gamma probe and subsequently removed; PA and pelvic lymphadenectomies, hysterectomy, and bilateral salpingo-oophorectomy followed. TUMIR was feasible in $90.5 \%$ of patients and showed a SLN identification in 55 patients $(74.3 \%)$. The sensitivity and NPV of SLN detected were, respectively, 92.3 and $97.7 \%$. No complications were registered. The advantages of TUMIR pointed by the authors are visualization of the exact injection site and puncture at the myometrial layer of the uterus; in this location, lymphatics are more significant compared to the subendometrial layer, allowing adequate information of the lymph node drainage of the endometrium. Disadvantages pointed are related with SLN detection failure associated with higher tumor size and technical skill requirements [26].

\section{Cervical injection}

Larger trials for SLN detection used cervical injection. Usually, a radioactive tracer is injected at two or four cardinal points in the cervix, the day preceding the surgery. Lymphoscintigraphy is then obtained. Immediately prior to surgery, a dye is injected at the same cervical sites.

The rate of detection ranges between 70 and $97 \%[3,8$, 27]. This is the most reproducible technique since the cervix is an accessible and easy site to perform the injection [16, 27]. It is also well tolerated by patients [24]. A potential concern with cervical injection is that it could represent more cervical drainage instead of corporal drainage [3, 9, 14]. SLNs are more often localized at the pelvis (93.1$100 \%$ [9]; the SLN detection of PA is lower than through hysteroscopic and myometrial injection, around $3 \%$ [9]. However, deep cervical injection at 3 and 9-o'clock positions prior to total hysterectomy may demonstrate good blue dye spread to the lower uterine segment and to the cornua of the uterus [14]. Besides, it is considered a good technique that shows a high bilateral pelvic detection rate [9], and cervical injection detects, on average, $63 \%$ of bilateral pelvic SLN against to 48 and $35 \%$ peritumoral and myometrial injections [9]. Holub et al. used a combined cervical and myometrial injection of blue dye and reported a SLN detection rate of $80 \%$ [31].

A French multicentric prospective trial published by Ballester et al. (SENTI-ENDO), involving 125 patients, used cervical dual labeling injection. Pelvic SLNs were detected in $89 \%$ of the patients (31\% unilateral and $69 \%$ bilateral). They explained this low detection rate with the long interval between radiocolloid injection and SLN procedure, with a median time of $22 \mathrm{~h}$ [11]. SLN in the PA region was detected in $5 \%$ of patients. For each hemipelvis as a unit, the presence of SLN was correctly identified in $100 \%$ of patients; however, at the patient level analysis, three patients with a type 2 EC had false-positive results, giving a sensitivity of $84 \%$ and a NPV of $97 \%$ [11].
In 2012, Barlin et al. published the largest prospective cohort, using a blue dye cervical injection. The surgical algorithm consisted in peritoneal washings and retroperitoneal evaluation with excision of all mapped SLN and suspicious nodes; in the absence of mapping, a side specific pelvis node dissection was performed. The overall SLN detection rate was $81 \%$ with optimal bilateral mapping in $51 \%$. There were seven false negatives, leading to a sensitivity of $85.1 \%$, a false-negative rate of $14.9 \%$, and NPV of $98.1 \%$; after algorithm application, the false-negative rate dropped to $2 \%$ [18].

Recently, How et al. published the first prospective data of 100 patients with EC undergoing SLN mapping. How et al. assessed SLN through a dual injection of Tc 99 and patent blue dye administrated during surgery, superficially in cervical submucosa and deeply in the cervix stroma. The surgical route was robot assisted, with a median time between incision and first blue node detection of $60.3 \mathrm{~min}$. SLN detection rate was $92 \%$, with a pelvic bilateral detection rate of $72 \%$ and PA level of $15 \%$. There was one false negative, leading to a sensitivity of $89 \%$ and a NPV of $99 \%$. During one procedure, How et al. noted in the infundibulo-pelvic ligament blue lymphatics, demonstrating that cervical injection could drain through this pathway [32]. Few studies evaluated directly the injection site with tumor localization and site of SLN identification.

Raspagliesi and Delaloye et al. in their series with hysteroscopic injection found no correlation between the site of SLN and site of the tumor [29, 37], stating that metastatic spread may not follow a stepwise progressive drainage [37]. According to Maccauro et al. and Niikura et al., the PA basin is frequently involved in drainage from the uterus corpus, and therefore, peri-tumoural injection allows a more complete detection of the corpus drainage [10] and becomes mandatory for assessing the PA area [28]. Also, subserosal injection could improve PA lymph node detection, considering its fundal drainage [33].

Only $16 \%$ of EC cancer is located in the lower part of the uterus [25], and cervical injection may miss higher pelvic or PA sentinel node identification [10]. That is why Holloway et al. recommended that should SLN not be identified with cervical injection, para-rectal and the pre-sacral space should be inspected, which may lead to identification of the common iliac or PA SLN [8]. Distribution of SLN and positive nodes according to the studies evaluating SLN in EC is found in Table 2.

Surgical route

The laparoscopic route seems to allow a higher SLN identification than laparotomy [6]. Mais et al. evaluated 34 patients in a prospective cohort with the purpose of comparing SLN detection in laparoscopy vs laparotomy. Cervical injection with blue dye was performed. Median time between injection and 
Table 2 Distribution of SLN and positive nodes according the studies evaluating SLN in EC

\begin{tabular}{|c|c|c|c|c|c|c|c|c|c|c|c|c|c|c|c|c|c|c|c|c|c|c|}
\hline $\begin{array}{r}\begin{array}{r}\text { Authors; site of } \\
\text { injection }\end{array} \\
\text { Anatomic location } \\
\text { of nodes }(\%)\end{array}$ & 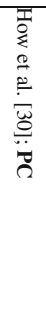 & 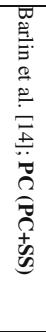 & 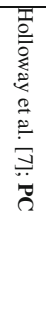 & 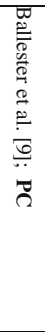 & 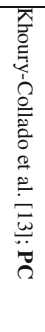 & 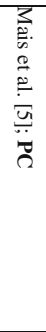 & 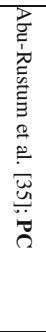 & 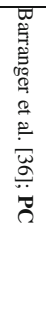 & 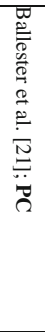 & 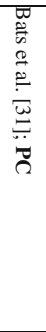 & 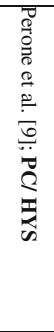 & 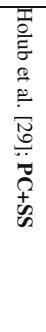 & 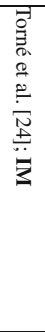 & 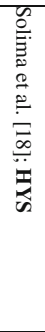 & 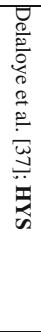 & 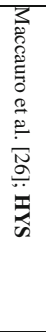 &  & 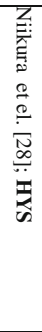 & 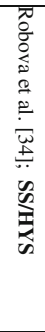 & $\begin{array}{l}5 \\
\stackrel{8}{0} \\
\infty \\
0 \\
0 \\
\stackrel{0}{0} \\
\Xi \\
\Xi \\
\mathscr{E}\end{array}$ & 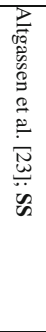 & 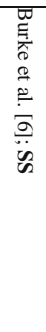 \\
\hline $\begin{array}{c}\text { Obturator } \\
\text { SLN detection } \\
\text { Positive SLN }\end{array}$ & NA & NA & NA & NA & NA & $\begin{array}{c}1 \\
\text { NA }\end{array}$ & $\begin{array}{r}24 \\
\text { NA }\end{array}$ & $\begin{array}{l}6 \\
1\end{array}$ & NA & NA & $\begin{array}{l}0,6 / \\
0,6 \\
0 / 1\end{array}$ & $\begin{array}{c}\mathrm{NA} \\
4\end{array}$ & $\begin{array}{r}25 \\
\text { NA }\end{array}$ & NA & $\begin{array}{c}20 \\
0\end{array}$ & $\begin{array}{c}14 \\
0\end{array}$ & $\begin{array}{r}15 \\
\text { NA }\end{array}$ & $\begin{array}{c}20 \\
0\end{array}$ & $\begin{array}{r}15 \\
\text { NA }\end{array}$ & $\begin{array}{r}17 \\
\text { NA }\end{array}$ & NA & $\begin{array}{r}13 \\
\text { NA }\end{array}$ \\
\hline $\begin{array}{c}\text { C. } \text { iliac } \\
\text { SLN detection } \\
\text { Positive SLN }\end{array}$ & NA & NA & NA & NA & NA & NA & $\begin{array}{c}8 \\
\text { NA }\end{array}$ & $\begin{array}{c}3 \\
\text { NA }\end{array}$ & $\begin{array}{c}6 \\
\text { NA }\end{array}$ & $\begin{array}{c}10 \\
0\end{array}$ & NA & $\begin{array}{r}18 \\
\text { NA }\end{array}$ & $\begin{array}{r}20 \\
\text { NA }\end{array}$ & $\begin{array}{c}18 \\
\text { NA }\end{array}$ & $\begin{array}{c}17 \\
0,5\end{array}$ & $\begin{array}{c}18 \\
1,5\end{array}$ & $\begin{array}{r}19 \\
\text { NA }\end{array}$ & $\begin{array}{l}6 \\
0\end{array}$ & $\begin{array}{r}10 \\
\mathrm{NA}\end{array}$ & $\begin{array}{c}3 \\
\text { NA }\end{array}$ & NA & $\begin{array}{r}19 \\
\text { NA }\end{array}$ \\
\hline $\begin{array}{c}\text { Ext. iliac } \\
\text { SLN detection } \\
\text { Positive SLN }\end{array}$ & NA & NA & NA & NA & NA & NA & $\begin{array}{r}30 \\
\text { NA }\end{array}$ & $\begin{array}{l}68 \\
11 \\
\end{array}$ & $\begin{array}{r}78 \\
\text { NA } \\
\end{array}$ & NA & NA & $\begin{array}{r}26 \\
\text { NA }\end{array}$ & $\begin{array}{r}27 \\
\text { NA }\end{array}$ & $\begin{array}{r}30 \\
\text { NA } \\
\end{array}$ & $\begin{array}{c}31 \\
0\end{array}$ & $\begin{array}{c}25 \\
3\end{array}$ & $\begin{array}{r}25 \\
\text { NA }\end{array}$ & $\begin{array}{c}25 \\
1\end{array}$ & $\begin{array}{r}59 \\
\text { NA } \\
\end{array}$ & $\begin{array}{r}25 \\
\text { NA }\end{array}$ & NA & NA \\
\hline $\begin{array}{c}\text { Int. iliac } \\
\text { SLN detection } \\
\text { Positive SLN }\end{array}$ & NA & NA & NA & NA & NA & NA & $\begin{array}{r}36 \\
\text { NA }\end{array}$ & $\begin{array}{l}0 \\
0\end{array}$ & NA & NA & NA & NA & $\begin{array}{c}1 \\
\text { NA }\end{array}$ & NA & $\begin{array}{c}18 \\
3\end{array}$ & $\begin{array}{l}3 \\
0 \\
\end{array}$ & $\begin{array}{c}4 \\
\text { NA }\end{array}$ & $\begin{array}{l}6 \\
0 \\
\end{array}$ & NA & $\begin{array}{r}19 \\
\text { NA }\end{array}$ & NA & $\begin{array}{c}3 \\
\text { NA }\end{array}$ \\
\hline $\begin{array}{c}\text { Interiliac } \\
\text { SLN detection } \\
\text { Positive SLN }\end{array}$ & NA & NA & NA & NA & NA & NA & $\begin{array}{l}0 \\
0\end{array}$ & $\begin{array}{c}20 \\
6\end{array}$ & $\begin{array}{r}16 \\
\text { NA }\end{array}$ & $\begin{array}{c}83 \\
9\end{array}$ & $\begin{array}{c}94 / 9 \\
1 \\
4 / 1\end{array}$ & NA & $\begin{array}{r}15 \\
\text { NA }\end{array}$ & $\begin{array}{r}31 \\
\text { NA }\end{array}$ & NA & NA & NA & NA & NA & NA & NA & $\begin{array}{c}7 \\
\text { NA }\end{array}$ \\
\hline $\begin{array}{l}\text { Parametrium } \\
\text { SLN detection } \\
\text { Positive SLN }\end{array}$ & NA & NA & NA & NA & NA & NA & $\begin{array}{l}0 \\
0\end{array}$ & $\begin{array}{l}0 \\
0\end{array}$ & $\begin{array}{l}0 \\
0\end{array}$ & $\begin{array}{l}0 \\
0\end{array}$ & 0 & $\begin{array}{l}0 \\
0\end{array}$ & $\begin{array}{l}0 \\
0\end{array}$ & NA & $\begin{array}{l}0 \\
0\end{array}$ & $\begin{array}{l}0 \\
0\end{array}$ & NA & NA & $\begin{array}{c}6 \\
\text { NA }\end{array}$ & NA & NA & $\begin{array}{l}0 \\
0\end{array}$ \\
\hline $\begin{array}{c}\text { Presacral } \\
\text { SLN detection } \\
\text { Positive SLN }\end{array}$ & NA & NA & NA & NA & NA & NA & $\begin{array}{l}0 \\
0\end{array}$ & NA & NA & $\begin{array}{l}7 \\
0\end{array}$ & 0 & NA & $\begin{array}{l}0 \\
0\end{array}$ & NA & $\begin{array}{l}0 \\
0\end{array}$ & NA & NA & $\begin{array}{l}1 \\
0\end{array}$ & $\begin{array}{c}2 \\
\text { NA }\end{array}$ & NA & $\begin{array}{l}2 \\
0\end{array}$ & NA \\
\hline $\begin{array}{c}\text { Pelvic } \\
\text { SLN detection } \\
\text { Positive SLN }\end{array}$ & $\begin{array}{c}91 \\
9\end{array}$ & NA & NA & $\begin{array}{l}95 \\
16\end{array}$ & 94 & NA & NA & NA & $\begin{array}{c}100 \\
25\end{array}$ & NA & NA & $\begin{array}{c}100 \\
4\end{array}$ & $\begin{array}{c}70 \\
7\end{array}$ & $\begin{array}{c}77 \\
4\end{array}$ & 86 & NA & NA & NA & NA & $\begin{array}{c}65 \\
1,6\end{array}$ & $\begin{array}{c}94 \\
4\end{array}$ & $\begin{array}{r}42 \\
\text { NA }\end{array}$ \\
\hline $\begin{array}{c}\text { PA } \\
\text { SLN detection } \\
\text { Positive SLN }\end{array}$ & $\begin{array}{l}9 \\
1\end{array}$ & NA & NA & $\begin{array}{l}5 \\
0\end{array}$ & 6 & NA & $\begin{array}{c}4 \\
\text { NA }\end{array}$ & $\begin{array}{l}0 \\
0\end{array}$ & $\begin{array}{l}0 \\
0\end{array}$ & $\begin{array}{l}0 \\
0\end{array}$ & $\begin{array}{c}0 / 18 \\
\text { NA }\end{array}$ & $\begin{array}{l}0 \\
0\end{array}$ & $\begin{array}{c}30 \\
1\end{array}$ & $\begin{array}{c}21 \\
2\end{array}$ & $\begin{array}{l}14 \\
0,5\end{array}$ & $\begin{array}{l}21 \\
1,5\end{array}$ & $\begin{array}{c}27 \\
1\end{array}$ & $\begin{array}{c}20 \\
0\end{array}$ & $\begin{array}{c}8 \\
\text { NA }\end{array}$ & $\begin{array}{c}35 \\
6\end{array}$ & $\begin{array}{l}6 \\
0\end{array}$ & $\begin{array}{r}39 \\
\text { NA }\end{array}$ \\
\hline
\end{tabular}

C-.Common; Ext.-External; HYS-Hysteroscopy; IM-Intra-myometrial guided transvaginal ultrasound; Int.-Internal; NA-Not available/applicable; PA-Para-aortic; PC-Peri-cervical/Cervical

pelvic lymph nodes dissection was 20-30 min with laparoscopic approach and 40-50 under laparotomy. SLN detection rate was $82 \%$ with laparoscopy, significantly higher than with laparotomy which was $42 \%$. Levenback et al. found, while mapping the cervix, that blue SLN was identifiable in the pelvis 5-16 min after injection and so remained for 9-30 min [7].

Globally, detection rates of SLN range between 44 and $100 \%[3,9,21]$. Achieving an acceptably low false-negative rate is crucial for lymphatic mapping as an alternative to standard protocols $[8,14]$. False-negative rates range between 0 and $33 \%$. SLN detection ranges from 0 to $92 \%$ with colorimetric techniques, 0 to $82 \%$ with isotopic, and 46 to $87 \%$ with dual techniques. In relation to the injection site, hysteroscopy has a detection rate of 50-100\%, cervical of $69-94 \%$, and myometrial of $67-92 \%$ [9]. A meta-analysis showed a SLN detection rate of $78 \%$, lower than for other solid tumors, with a sensitivity of $95 \%$. In this meta-analysis, cervical injection was correlated with an increase of the detection rate, hysteroscopic injection was associated with a decrease of detection rate and myometrial injections with a decrease of sensitivity [18]. PA node evaluation is less studied. Burke et al. found 31 SLN, and 12 of them were in the PA area. Niikura et al. detected SLN in $82 \%$ of patients, with at least 1 PA SLN in 18 of 25 patients, and SLN located in the PA area in 3 of 23 patients [30]. Delpesh et al. reported a lower detection rate of SLN in the PA region using cervical injection alone compared with cervical combined with subserosal or peritumoral injections. Peritumoral or subserosal myometrial injection may enable detection of isolated PA node involvement. In cervical injection studies, PA nodes were not systematically sampled. SENTI-ENDO identified 5 in 111 with an associated SLN in the PA region. Detection of isolated PA node involvement would improve outcome prediction and may decrease the complications of postoperative whole pelvis radiotherapy by limiting the use of extended surgical staging [33].

\section{Ultrastaging}

Lymph node status is an important factor and a criterion for adjuvant therapy in EC [9]. Ultrastaging of lymph nodes is a main focus of the SLN concept and implies serial sectioning and IHC $[11,34]$. The main limitation of these techniques is their time-consuming and costly nature, inappropriate for routine use [3, 34].

At the Philadelphia Consensus Conference, macrometastasis was defined as a single focus of metastastic disease per node 
measuring more than $2 \mathrm{~mm}$, micrometastasis measuring between 0.2 and $2 \mathrm{~mm}$ and submicrometastasis measuring less than $0.02 \mathrm{~mm}$, including the presence of a single noncohesive tumor cell $[9,34]$.

The relation between micrometastasis and risk of recurrence/prognosis has been demonstrated in an increasing number of malignancies suggesting that micrometastasis should be an indication for adjuvant therapy, including early EC [34]. Yabushita et al. analyzed the relation between disease recurrence and presence of pelvic nodes micrometastasis in early $\mathrm{EC}$ and found its presence was associated with recurrent disease [34].

The conventional examination of SLN with hemi-section and analysis under hematoxylin and eosin (HE) is not very effective for micrometastasis detection [9]. Ultrastaging protocols involve serial sectioning techniques (with intervals of $3 \mathrm{~mm}$ ), and each of these sections will be analyzed into a panel of anti-cytokeratin antibodies [9, 34]. The signal amplification produced by IHC may improve sensitivity of micrometastasis detection [32]. The rate of detection of micrometastasis ranged from 0 to $15 \%$ according to a review [34]. Niikura et al., using serial sectioning and IHC, noted that micrometastases were detected in $5 \%$ negative SLN and only in $0.3 \%$ of nonsentinel nodes [30]. Ballester et al. showed that ultrastaging detected metastasis underdiagnosed by conventional histology in $11 \%$ and also showed that SLN biopsy upstaged $10 \%$ of low-risk and $15 \%$ of intermediate-risk EC patients [11]. Holloway et al. concluded that metastasis was solely identified by ultrastaging and IHC in 4/10 patients with node metastasis, which represents a $67 \%$ increase in identification of node metastasis compared to routine HE [8]. Khoury-Collado et al. found metastasis only detected by ultrastaging protocols in $3 \%$ of patients and that SLNs are more prone to be metastatic than non SLNs [35].

\section{Conclusions}

Patients with gynecological malignancies, particularly with small tumors of the vulva, have significantly profited from SLN mapping. Since its first application in 1996, SLN in EC is still debated. Considering the surgical risk of EC patients, the lower risk of metastasis in early stages and the controversial role of therapeutic lymphadenectomy in unselected patients, SLN biopsy, incorporating ultrastaging, could find its indication in low-and intermediate-risk women with EC: it brings the advantages of a conservative and more sensitive procedure and might select patients for adjuvant therapy. Moreover, SLN biopsy lends itself to laparoscopic surgery, which is an attractive alternative [10, 19].

The uterus has a complex lymphatic drainage due to its midline position, and therefore, the best technique for lymphatic ways highlighting remains in discussion. Data on
SLN in EC are very heterogeneous in methodology and studied population. Injection site is the most discussed issue. Subserosal myometrial injection has considerable variability in SLN detection and lower sensitivity; hysteroscopic injection has demonstrated the highest detection rates; however, it adds costs and is technically demanding; cervical injection is more reproducible, yet the least reliable in PA mapping. A meta-analysis estimated a detection rate of SLN in EC of $78 \%$, lower than for other solid tumors. Recent larger studies, with more consistent results and higher detection rates, point the SLN procedure as feasible, reliable, and easy to incorporate in surgical management.

In the future, cost-effectiveness of potential benefits from SLN procedure compared with adjuvant therapies should be evaluated in low- and intermediate-risk women with EC. Also, larger, prospective and controlled trials are needed to evaluate the most reproducible and effective standardized procedure to detect SLN in EC.

Acknowledgments We thank to João Santos, MD.

Statement of responsibility All authors gave substantial contributions to the article on reviewing recent literature and actively participating on its elaboration. The authors approved the final version of the manuscript.

Conflict of interest The authors declare that they have no competing interests.

\section{References}

1. Garg G, Gao F, Wright J (2013) The risk of lymph node metastasis with positive peritoneal cytology in endometrial cancer. Int $\mathrm{J}$ Gynecol Cancer 23:90-97. doi:10.1097/ICG.0b013e318275afd2

2. Dogan NU, Gungor T et al (2011) To what extent should paraaortic lymphadenectomy be carried out for surgically staged endometrial cancer? Int J Gynecol Cancer 22:607-610. doi:10.1097/ IGC.0b013e3182434adb

3. Lecuru F, Bats S et al (2012) Technique et résultats du prélèvement $\mathrm{du}$ ganglion sentinelle dans le cancers du col et du corps de l'utérus. EMC. doi:10.1016/S1624-5857(12)57321-7

4. AlHilli MM, Mariani A (2013) The role of para-aortic lymphadenectomy in endometrial cancer. Int J Clin Oncol. doi:10.1007/ s10147-013-0528-7, Article online

5. Amos C, Blake P et al (2009) Efficacy of systematic pelvic lymphadenectomy in endometrial cancer (MRC ASTEC trial): a randomised study. Lancet 373(9658):125-136. doi:10.1016/ S0140-6736(08)61766-3

6. Mais V, Peiretti M et al (2010) Intraoperative sentinel lymph node detection by vital dye through laparoscopy or laparatomy in early endometrial cancer. J Surg Oncol 101:408-412. doi:10.1002/ jso. 21496

7. Burke TW, Levenbeck $C$ et al (1996) Intraabdominal lymphatic mapping to direct selective pelvic and paraaortic lymphadenectomy in women with high-risk endometrial cancer: results of a pilot study. Gynecol Oncol 62:169-173

8. Holloway RW, Bravo RAM et al (2012) Detection of sentinel lymph nodes in patients with endometrial cancer undergoing robotic- 
assisted staging: a comparison of colorimetric and fluorescence imaging. Gynecol Oncol Article in Press. doi:10.1016/j.ygyno. 2012.04.009

9. Bonneau C, Bricou A, Barranger E (2011) Current position of the sentinel lymph node procedure in endometrial cancer. Bull Cancer 98(2):133-145. doi:10.1684/bdc.2011.1304

10. Perone AM, Casadio P et al (2008) Cervical and hysteroscopic injection for identification of sentinel lymph node in endometrial cancer. Gynecol Oncol 111:62-67. doi:10.1016/j.ygyno.2008.05.032

11. Ballester M, Dubernard G et al (2011) Detection rate and diagnostic accuracy of sentinel-node biopsy in early stage endometrial cancer: a prospective multicentric study (SENTI-ENDO). Lancet Oncology 12:469-476. doi:10.1016/S14702045(11)70070-5

12. Lopes LAF, Nicolau SM et al (2007) Sentinel lymph node in endometrial cancer. Int J Gynecol Cancer 17:1113-1117. doi:10.1111/j.1525-1438.2007.00909.x

13. Lelievre L, Camatte S et al (2004) Sentinel lymph node biopsy in cervix and corpus uteri cancers. Int J Gynecol Cancer 14:271-278

14. Khoury-Collado F, Abu-Rustum NR (2008) Lymphatic mapping in endometrial cancer: a literature review of current techniques and results. Int J Gynecol Cancer 18:1163-1168. doi:10.1111/j.1525-1438.2007.01188.x

15. Fujimoto T, Fukuda J, Tanaka T (2007) Role of complete paraaortic lymphadenectomy in endometrial cancer. Curr Opin Obstet Gynecol 21:10-14. doi:10.1097/GCO

16. Nasuh D, Gungor T et al (2012) To what extend should PA lymphadenectomy be carried out for surgically staged endometrial cancer. Int J Gynecol Cancer 22:607-610. doi:10.1097/ IGC.0b013e3182434adb

17. Bouquier J, Bricou A, Delpech Y (2010) Y a-t-il un intérêt au curage lomboartique dans les cancers de l'endomètre opérables. Bulletin du Cancer 97(2):199-207. doi:10.1684/bdc.2009.0956

18. Barlin JN, Khoury-Collado F et al (2012) The importance of applying a sentinel lymph node mapping algorithm in endometrial cancer staging: beyond removal of blue nodes. Gynecol Oncol Article in Press. doi:10.1016/j.ygyno.2012.02.021

19. Kitchener HC (2011) Sentinel-node biopsy in endometrial cancer: a win-win scenario? Lancet Oncol 12:469-470. doi:10.1016/ S14702045(11)70093-6

20. Kang S, Yoo HJ et al (2011) Sentinel lymph node biopsy in endometrial cancer: meta-analysis of 26 studies. Gynecol Oncol 123:522-527. doi:10.1016/j.ygyno.2011.08.034

21. Solima E, Martinelli F et al (2012) Diagnostic accuracy of sentinel node in endometrial cancer by using hysteroscopic injection of radiolabeled tracer. Gynecol Oncol 126:419-423. doi:10.1016/ j.ygyno.2012.05.025

22. Rossi EC, Ivanova A, Boggess JF (2012) Robotically assisted fluorescence-guided lymph node mapping with ICG for gynecologic malignancies: a feasibility study. Gynecol Oncol 124(1):7882. doi:10.1016/j.ygyno.2011.09.025
23. Ballester M, Dubernard G et al (2008) Use of the sentinel node procedure to stage endometrial cancer. Ann Surg Oncol 5:15231529. doi:10.1245/s10434-008-9841-1

24. Ballester M, Rouzier R et al (2009) Limits of lymphoscintigraphy for sentinel node biopsy in women with endometrial cancer. Gynecol Oncol 112(2):348-352. doi:10.1016/j.ygyno.2008.11.004

25. Altgassen C, Pagenstecher J et al (2007) A new approach to label sentinel nodes in endometrial cancer. Gynecol Oncol 105(2):457-461. doi:10.1016/j.ygno.2007.01.021

26. Torné A, Pahisa J et al (2012) Transvaginal ultrasound-guided myometrial injection of radiotracer (TUMIR): a new method for sentinel lymph node detection in endometrial cancer. Gynecol Oncol. doi:10.1016/j.ygyno.2012.10.008

27. Dubernard G, Darai E, Ballester M (2012) Arguments in favour of sentinela lymph node dissection in endometrial cancer. Gynécologie Obstétrique \& Fertilité 40:261-263. doi:10.1016/j.gyobfe. 2012.02.005

28. Maccauro M, Lucignani G et al (2005) Sentinel lymph node detection following the hysterocopic peitumoral injection of $99 \mathrm{mTc}-$ labelled albumin nanocolloid in endometrial cancer. Eur J Nucl Med Mol Imaging 32(5):569-574. doi:10.1007/s00259-004-1709-4

29. Raspagliesi F, Ditto A et al (2004) Hysteroscopic injection of tracers in sentinel node detection of endometrial cancer: a feasibility study. Am J Obstet Gynecol 191(2):435-439. doi:10.1016/j.ajog.2004.03.008

30. Niikura $H$, Okamura $C$ et al (2004) Sentinel lymph node detection in patients with endometrial cancer. Gynecol Oncol Feb:92(2):669-674. doi:10.1016/j.ygyno.2003.10.039

31. Holub Z, Jabor A et al (2004) Laparoscopic detection of sentinel lymph nodes using blue dye in women with cervical and endometrial cancer. Med Sci Monit 10(10):CR587-CR591

32. How J, Lau S et al (2012) Accuracy of sentinel lymph node detection following intra-operative cervical. Gynecol Oncol 127(2012):332-337. doi:10.1016/j.ygyno.2012.08.018

33. Bats AS, Clement D et al (2008) Does sentinel node biopsy improve the management of endometrial cancer? Data from 43 patients. J Surg Oncol 97(2):141-145. doi:10.1002/jso.20857

34. Bézu C, Coutant $C$ et al (2010) Ultrastaging of lymph node in uterine cancers. J Exp Clin Cancer Res 29(5):1-8

35. Khoury-Collado F, Murray MP et al (2011) Sentinel lymph node mapping for endometrial cancer improves the detection of metastatic disease to regional lymph nodes. Gynecol Oncol 122:251254. doi:10.1016/j.ygyno.2011.04.030

36. Robova H, Charvat M et al (2009) Lymphatic mapping in endometrial cancer: comparison of hysteroscopic and subserosal injection and the distribution of sentinel lymph nodes. Int $\mathrm{J}$ Gynecol Cancer 19(3):91-394. doi:10.111/IGC.0b013e3181a1c0b1

37. Delaloye J-F, Pampallona S et al (2007) Intraoperative lymphatic mapping and sentinel node biopsy using hysteroscopy in patients with endometrial cancer. Gynecol Oncol 106:89-93. doi:10.1016/ j.ygyno.2007.03.003 